\title{
Potentiale und Risiken der Nutzung von Methan aus Methanhydraten als Energieträger
} Groth, Markus

Publication date:

2009

Document Version

Verlags-PDF (auch: Version of Record)

Link to publication

Citation for pulished version (APA):

Groth, M. (2009). Potentiale und Risiken der Nutzung von Methan aus Methanhydraten als Energieträger. (Working Papers Series in Economics; Nr. 147). Institut für Volkswirtschaftslehre der Universität Lüneburg.

\section{General rights}

Copyright and moral rights for the publications made accessible in the public portal are retained by the authors and/or other copyright owners and it is a condition of accessing publications that users recognise and abide by the legal requirements associated with these rights.

- Users may download and print one copy of any publication from the public portal for the purpose of private study or research.

- You may not further distribute the material or use it for any profit-making activity or commercial gain

- You may freely distribute the URL identifying the publication in the public portal ?

Take down policy

If you believe that this document breaches copyright please contact us providing details, and we will remove access to the work immediately and investigate your claim. 
Potentiale und Risiken der Nutzung von Methan aus Methanhydraten als Energieträger

\author{
von \\ Markus Groth
}

University of Lüneburg Working Paper Series in Economics

No. 147

Oktober 2009

www.leuphana.de/vwl/papers

ISSN 1860 - 5508 


\title{
Potentiale und Risiken der Nutzung von Methan aus Methanhydraten als Energieträger
}

\author{
Markus Groth $^{1}$
}

\begin{abstract}
Marine and permafrost-based methane hydrates are the largest existing fossil carbon resource, whereby the marine deposits far outweigh the terrestrial ones. Their broad geographic distribution, especially in comparison to oil and conventional gas, make them a promising future source of energy. However, there is a danger of forcing the greenhouse effect in the event of a release of methane into the atmosphere as well as causing the collapse of oceanic slope sediments. Also the technical difficulties in extracting methane from hydrates are not yet fully resolved. Nevertheless, research on methane hydrates has been forced both on political as well as economic considerations in recent years and methane hydrates have several practical advantages, which make them a transitional solution worth looking at on the way to a future renewable-based energy supply, not in the least in playing a role in carbon capture and sequestration. However, the knowledge of the potentials and risks of methane hydrates is still very poor, especially in the German-speaking public, administration and policies. This deficiency hopefully will be eased by this overview dealing with the current state of research and an outlook based on the most important findings.
\end{abstract}

\section{Schlüsselwörter}

$\mathrm{CO}_{2}$-Sequestrierung, Energiepolitik, Erdgas, fossile Energieträger, Klimawandel, Kohlenstoffpotential, Methan, Methanhydrate, Ökologische Ökonomik, Treibhausgas, Versorgungssicherheit

\section{JEL-Klassifikation}

H00, Q38, Q47, Q42, Q48, Q54

\footnotetext{
${ }^{1}$ Dr. Markus Groth, Leuphana Universität Lüneburg, Lehrstuhl für Nachhaltigkeitsökonomie, Scharnhorststr. 1, D-21335 Lüneburg, E-Mail: groth@uni-lueneburg.de, Tel.: 04131 / 677-2636, Fax.: 04131 / 677-1381.
} 


\section{Einleitung}

In den vergangenen Jahren wurde die Suche nach Alternativen zu den konventionellen Energieressourcen Erdöl, Erdgas und Kohle erheblich intensiviert. Diese Entwicklung wurde maßgeblich durch die gestiegenen Rohstoffpreise wichtiger Energieträger, die zunehmende Verknappung fossiler Ressourcen, globale Klimaveränderungen und die instabile Sicherheitslage in vielen bedeutenden Rohstoff exportierenden Staaten forciert (World Energy Council 2007). Im Zuge dessen sind neben den erneuerbaren Energieträgern auch Methanhydrate zunehmend in den Fokus getreten. Ihr Kohlenstoffvorkommen wurde Ende des vergangenen Jahrhunderts weltweit auf bis $\mathrm{zu} 10.000 \mathrm{Gt} \mathrm{C}$ in Form von Methankohlenstoff geschätzt. Diese Quantifizierung von Methanhydraten ist zweifelsohne mit großen Unsicherheiten verbunden und die Vorhersagen wurden beständig nach unten korrigiert. Jüngere und gleichsam konservativere Schätzungen gehen global von bis zu 3.000 Gt C in Methanhydraten aus (Rogner 1997; Kvenvolden und Lorenson 2001; Buffet und Archer 2004; Milkov 2004; Archer 2005). Die Kohlenstoffgehalte der unter heutigen Bedingungen ökonomisch abbaubaren Reserven betragen für Kohle 675 Gt C, für Erdöl 160 Gt C und für Erdgas 96 Gt C (EIA 2007).

Selbstverständlich ist bei diesem Vergleich $\mathrm{zu}$ berücksichtigen, dass hier die unter heutigen Bedingungen ökonomisch abbaubaren Ressourcen für Kohle, Erdöl und Erdgas den gesamten Ressourcen der Methanhydrate gegenübergestellt werden. Die zu heutigen Bedingungen ökonomisch abbaubaren Ressourcen von Methanhydraten sind gleich Null. Somit ist diese Gegenüberstellung nicht als Vergleich zwischen den verschiedenen fossilen Energieträgern $\mathrm{zu}$ verstehen, sondern sie soll dazu dienen, die Größenordnung des Kohlenstoffpotentials von Methanhydraten und ihre $\mathrm{zu}$ erwartende Bedeutung für die zukünftige Energieversorgung zu verdeutlichen.

Hinsichtlich der Entwicklung des weltweiten Energieverbrauchs prognostiziert die Internationale Energie Agentur (IEA) im Referenzszenario für das Jahr 2030 einen Bedarf von rund 17,7 Mrd. Tonnen Rohöleinheiten. Dies entspricht gegenüber dem Jahr 2005 einem jährlichen Anstieg der Energienachfrage von rund 1,8\% und einem Gesamtzuwachs von ungefähr 55\%. Im Zuge dessen wird erwartet, dass die konventionellen fossilen Ressourcen einen Anteil von rund 84\% des Verbrauchsanstiegs decken werden (IEA 2007b).

Unbestritten ist der menschliche Einfluss auf den fortschreitenden Klimawandel. So wurden von der industriellen Revolution bis zum Beginn des 21. Jahrhunderts - je nach Studie und Berechnungsgrundlage - zwischen 277 und 315 Gt Kohlenstoff aus fossilen Energieträgern verbrannt und entsprechend Kohlendioxid $\left(\mathrm{CO}_{2}\right)$ emittiert (World Energy 
Council 2000; Marland et al. 2005; World Resources Institute 2005). Dies hat zu einem Anstieg der Treibhausgaskonzentration in der Atmosphäre von $280 \mathrm{ppm}^{2}$ auf 380 ppm $\mathrm{CO}_{2}$ und einem Temperaturanstieg von im Mittel $14^{\circ} \mathrm{C}$ auf $15^{\circ} \mathrm{C}$ geführt (IPCC 2007a; IPCC 2007b).

Um einem weiteren Anstieg der Konzentration von Treibhausgasen entgegenzuwirken, steht die globale Energieversorgung vor entscheidenden Weichenstellungen (Stern 2006; IPCC 2007a). Im Zuge dessen ist auch die Herausforderung zu berücksichtigen, der weltweit in der Summe steigenden Nachfrage nach Energie unter ökonomischen und ökologischen Gesichtspunkten nachhaltig gerecht zu werden.

Doch welche Rolle wird in diesem Zusammenhang die Nutzung von Methanhydraten einnehmen? Das Wissen über die damit verbundenen Potentiale und Risiken ist insbesondere in der deutschsprachigen Öffentlichkeit und Politik noch immer gering ausgeprägt. Diesem Mangel wird nachfolgend durch eine Analyse des aktuellen Stands der Forschung entgegengewirkt. Hierzu wird insbesondere diskutiert, welchen Beitrag die Nutzung von Methanhydraten im Rahmen der zukünftigen globalen Energieversorgung sehr wahrscheinlich leisten kann. Im Zuge dessen wird auch das grundsätzliche Potential einer Kombination der Methangewinnung aus Hydraten mit der Sequestrierung von $\mathrm{CO}_{2}$ aufgezeigt.

Im zweiten Abschnitt werden grundlegende Aspekte zu Methanhydraten eingeführt, es wird ein Überblick über die bisher nachgewiesenen Methanhydratvorkommen gegeben und das Risikopotential der menschlichen Nutzung von Methanhydraten skizziert. Abschnitt Drei diskutiert den aktuellen Stand der Erprobung und Nutzung von Methanhydraten als Energieträger. Im vierten Abschnitt wird die Möglichkeit einer Kopplung des Abbaus von Methanhydraten mit der gleichzeitigen Sequestrierung von $\mathrm{CO}_{2}$ vorgestellt, bevor in Abschnitt Fünf ein zusammenfassendes Fazit unter Einbeziehung noch offener Forschungsfragen den Beitrag abschließt.

\section{Grundlagen, Methanhydratvorkommen und Risiken der Förderung von Methan aus}

\section{Methanhydraten}

Methan $\left(\mathrm{CH}_{4}\right)$ ist wie Erdgas und Erdöl ein kohlenstoffhaltiger und fossiler Energieträger, wobei Methangas bei seiner Verbrennung auch Kohlendioxid freisetzt und überwiegend in Gemischen mit anderen Gasen als Brenngas verwendet wird (Kvenvolden 2003). Methan gehört zu den Spurengasen und ist nach Kohlendioxid das zweitwichtigste Treibhausgas. Es entsteht bei organischen Gär- und Zersetzungsprozessen und ist Hauptbestandteil sowohl von

${ }^{2} \mathrm{ppm}=$ parts per million. 
Erdgas als auch Biogas. Gelangt Methan in die Atmosphäre, wirkt es dort über einen Zeitraum von 100 Jahren je Molekül rund 25-mal stärker als Kohlendioxid und kann durch seine Freisetzung gravierend zum Treibhauseffekt beitragen (Buffett 2000; Max und Pellenbarg 2003; IPCC 2007a; IFM-GEOMAR 2007).

Methanhydrate sind feste bzw. eisartige molekulare Verbindungen zwischen Wasser und Gas, die ausschließlich bei bestimmten Druck-Temperatur-Bedingungen stabil sind (Carroll 2003; Kvenvolden 2003; Archer 2005; USGS 2006). Bei Methanhydraten bauen die Wassermoleküle Käfigstrukturen auf, in denen die Gasmoleküle eingeschlossen sind. Sie werden deshalb auch als Einschlussverbindungen oder Clathrate bezeichnet, denn Wasser und Methan gehen keine direkte chemische Bindung ein. Stattdessen lagert sich das Methan in Hohlräumen innerhalb des Kristallgitters des Eises ab (Sloan 1998a; Carroll 2003).

Methanhydrate weisen hinsichtlich ihrer Stabilitätsbedingungen spezifische Eigenschaften auf, die sie grundlegend von anderen Energieträgern unterscheiden (Torres et al. 2004; Wallmann et al. 2006). An Land existieren die notwendigen Druck-Temperatur-Bedingungen ausschließlich in den Permafrostgebieten (vor allem auf der Nordhalbkugel) während die Hydratvorkommen in den Ozeanen weltweit verteilt sind (Kvenvolden und Lorenson 2001; Milkov 2004).

In den Permafrostgebieten finden sich Hydratvorkommen ab einer Tiefe von 150 Metern unter der Oberfläche. Dabei reicht die Stabilitätszone auf etwa 2000 Meter Tiefe hinab. Die Stabilitätszonenen in den Ozeanen beginnen in der Regel ab Tiefen von etwa 300 Metern, da erst dort ein entsprechender Druck die Stabilität garantiert. Als Ausnahme gelten die polaren Schelfgebiete, in denen ein vergleichsweise niedriger Druck durch konstant niedrige Temperaturen ausgeglichen wird. Hier konnten Methanhydratvorkommen bislang ab einer Tiefe von 100 - 150 Metern nachgewiesen werden (Max und Pellenbarg 2003). Das untere Limit der Stabilitätszonen ergibt sich aus den geothermischen Eigenschaften im Erdboden. Zwar kompensiert der zunehmende Druck die Temperaturzunahme, kann dies aber ab einem bestimmten Punkt nicht mehr ausgleichen. Folglich sind die Stabilitätszonen häufig nur mehrere hundert Meter dick. Darunter kann das Methan wieder gasförmig vorkommen (Sloan 1998; Kvenvolden 2003; Hornbach et al. 2004; WBGU 2006).

Methanhydrate sind imstande große Mengen Gas zu speichern. Ein Volumen Hydrat enthält rund 170 Volumina Methan unter Standardbedingungen - also bei Luftdruck und $25^{\circ} \mathrm{C}$ (WBGU 2006). Die Bewertung der weltweit in Hydraten gebundenen Methanmengen ist noch immer mit großen Unsicherheiten verbunden. Die bisherigen Schätzungen schwanken in einem Bereich von 500 bis 10.000 Gt Kohlenstoff (Kvenvolden 1988; Rogner 1997; 
Kvenvolden und Lorenson 2001; Collett 2003; Kvenvolden 2003; Buffet und Archer 2004; Milkov 2004; Archer 2005). Hierbei ist zu erkennen, dass die anfangs sehr optimistischen Prognosen zunehmend angepasst wurden und die jüngsten Untersuchungen eine Größenordnung von bis zu 3.000 Gt Kohlenstoff in Methanhydraten ausweisen.

Weltweit wurden bislang rund 20 Vorkommen von Methanhydraten über direkte Beprobungen nachgewiesen. Weitere 80 Standorte wurden anhand von geochemischen, geophysikalischen und geologischen Anhaltspunkten lokalisiert (Kvenvolden und Lorenson 2001; Milkov 2004; Max et al. 2006). Größere Gashydratfelder existieren in Nordrussland, in den arktischen Regionen von Nordamerika sowie auf verschiedenen Inseln der Arktis. Im Meeresboden wurden Vorkommen im Golf von Mexiko, dem Schwarzen und Kaspischen Meer, dem Eel River Becken vor Kalifornien und dem Ochotskischen Meer entdeckt (U.S. Department of Energy 2006). Weiterhin finden sich größere Felder vor der Küste Perus, im nördlichen Teil des Südchinesischen Meeres, im indischen Krishna-Godavari-Becken, vor der Pazifikküste Japans und im Nankai Becken sowie im Ulleuung Becken (Collett 2003; Buffett und Archer 2004; Zhang et al. 2007b; Park et al. 2008; Research Consortium for Methane Hydrate Resources in Japan 2008). Einen weltweiten Überblick über die bisher lokalisierten Methanhydratfunde gibt Abbildung 1.

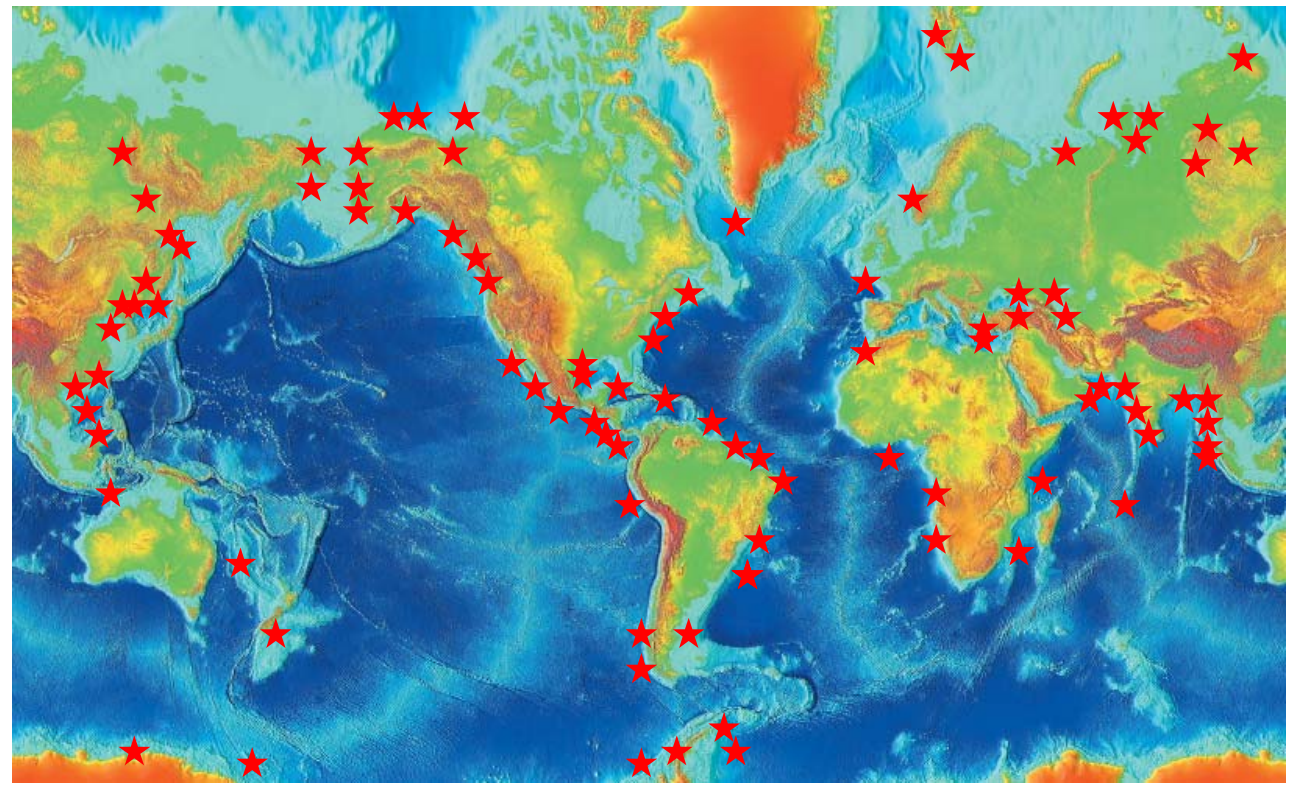

Abb. 1: Weltweite Methanhydratfunde. Quelle: Eigene und aktualisierte Darstellung, basierend auf Kvenvolden und Lorenson (2001). Die Weltkarte wurde durch das NOAA's National Geophysical Data Center (NGDC) unter http://www.ngdc.noaa.gov/mgg/fliers/00mgg05.html bereitgestellt. 
Im Verlauf der Prozesskette aus Prospektion, Exploration, Quantifizierung, Abbau und Transport von Methangas aus Hydraten sind mit jedem Schritt Umweltrisiken verbunden. ${ }^{3}$ Hierbei stehen Risiken einer Freisetzung von Treibhausgasen sowie durch eine Destabilisierung der Sedimente ausgelöste untermeerische Hangrutschungen im Mittelpunkt (Wallmann 2007). Methan ist nach Kohlendioxid das bedeutendste Treibhausgas, wobei mit seiner industriellen Nutzung die nachfolgenden Risiken verbunden sind (Kennett et al. 2003).

Während Methan in den Ozeanen über eine Lebensdauer von etwa 50 Jahren verfügt, oxidiert es in der Atmosphäre nach rund 8 Jahren zu Kohlendioxid. Dadurch schwächt sich die Wirkung ab, jedoch weist Kohlendioxid eine weitaus längere Lebensdauer als Methan auf und ein großer Austritt des Gases hätte langfristige Auswirkungen auf den Treibhauseffekt (Kvenvolden 1988; Dickens 2004). Zusätzlich besteht die Gefahr, dass sich die Methan- und Kohlendioxidkonzentration in den Ozeanen erhöht, was den Prozess der Versauerung weiter verstärken würde (WBGU 2006).

Ein zentrales Risiko bei der industriellen Förderung von Methanhydraten ist somit dahingehend vorhanden, dass sich eine beim Abbau verursachte ungeplante Destabilisierung kaum steuern ließe und sie massive Methanfreisetzungen nach sich ziehen würde.

Zudem ist bei einem Abbau von Methanhydraten an den Kontinentalhängen ihr stabiler Verbund mit den örtlichen Sedimenten zu berücksichtigen. Diese Methanhydrate haben einen signifikanten Einfluss auf die Stabilität des gesamten Hangs, da sie im Porenraum von marinen Sedimenten wie Zement wirken (Archer 2005). Wenn sich die Stabilitätsbedingungen vor Ort durch menschliche Eingriffe verändern, zerfallen die Hydrate zu Wasser und freiem Gas. Eine solche Anreicherung von Flüssigkeit und Gas in den Sedimentschichten macht die Hänge instabil und kann eine vollständige Destabilisierung begünstigen sowie submarine Rutschungen mit der Folge von Tsunami-Wellen nach sich ziehen (Driscoll et al. 2000; USGS 2006).

\section{Stand der Erprobung und Perspektiven der Nutzung von Methan aus Methanhydraten}

Die Erforschung von Methanhydraten in Permafrostgebieten ist relativ weit vorangeschritten, wobei hinsichtlich der Fördertechniken insbesondere Erfahrungen durch Projekte am Gasfeld Messoyakha in Sibirien und dem Forschungsprojekt Mallik in Kanada gesammelt werden

\footnotetext{
${ }^{3}$ Die Frage der Zugriffstechnik zur Destabilisierung und zum Abbau von Methanhydraten wird in Abschnitt Vier aufgegriffen. Hierbei wird insbesondere auf die unter Berücksichtigung von Klimaschutzaspekten innovativste Zugriffstechnik der Injektion von flüssigem $\mathrm{CO}_{2}$ zur Destabilisierung der Methanhydrate bei einer gleichzeitigen Sequestrierung von Kohlendioxid eingegangen.
} 
konnten (Collett 2003; Buffett und Archer 2004). Das Forschungsprojekt Mallik gilt als eines der ambitioniertesten Projekte und wird im Mackenzie Delta vor der arktischen Küste Kanadas durchgeführt. Ein internationaler Forschungsverbund - bestehend aus den USA, Kanada, Japan, Indien und Deutschland - initiierte das Projekt, bei dem Produktionstests im Mittelpunkt stehen (Technology Research Center Japan National Oil Corporation 2003; Dallimore 2007).

Das erste staatliche Programm wurde 1982 in den USA durch das Energieministerium aufgelegt und im Jahr 1992 aufgrund der niedrigen Preise für konventionelle Rohstoffe sowie geänderter politischer Prioritäten eingestellt. Seit dem Jahr 2000 wurde die Forschungstätigkeit seitens des Energieministeriums durch das im Rahmen des Methane Hydrate Research and Development Act eingeführte National Methane Hydrate Program wieder aufgenommen. Im Zuge dessen wurden bis zum Jahr 2005 jährlich rund 9 Millionen Dollar in die Erforschung von Methanhydraten investiert (Federal Methane Hydrate Advisory Committee 2007). Das Forschungsprogramm wurde im August 2005 durch den Energy Policy Act bis zum Jahr 2010 verlängert, wobei das spezifische Budget des Energieministeriums auf rund 12 Millionen Dollar pro Jahr erhöht wurde (WBGU 2006; Federal Methane Hydrate Advisory Committee 2007). Die Erfüllung der innerhalb des Methane Hydrate Research Program festgeschriebenen Ziele soll durch die im Juli 2006 verabschiedete Interagency Roadmap for Methane Hydrate Research and Development erreicht werden. Die technische Realisierbarkeit wird darin grundsätzlich bereits für die Permafrostböden in Alaska festgestellt, wobei eine allumfassende ökologische, technische und ökonomische Bewertung dieser Vorkommen in ihrer kommerziellen Nutzung ab dem Jahr 2015 resultieren soll. Als Meilenstein für die Erschließung mariner Vorkommen wird das Jahr 2025 genannt, wobei die Methanhydratvorkommen im Golf von Mexico im Fokus stehen (U.S. Department of Energy 2006).

Große Erfolge bei der Erkundung von marinen Gashydraten hat China im Jahr 2007 erzielen können (Zhang et al. 2007a). Im Rahmen einer ersten Expedition mit dem Ziel der Bebohrung von acht vermuteten Methanhydratvorkommen in Wassertiefen von bis zu 1500 Metern im nördlichen Teil des Südchinesischen Meeres (innerhalb des Gebietes Shenhu) wurden in drei Vorkommen Gashydrate nachgewiesen. Innerhalb der 10 bis 25 Meter dicken Lagerstätten ist Methan mit einem Anteil von über 99\% das dominierende Gas. Nicht zuletzt ermuntert durch 
diese viel versprechenden Ergebnisse werden weitere Expeditionen in unterschiedlichen Gebieten im nördlichen Teil des Südchinesischen Meeres diskutiert (Zhang et al. 2007b).

Indien ist zusammen mit China der am schnellsten wachsende Verbraucher von fossilen Energieträgern (IEA 2007a). Um der damit verbundenen hohen Importabhängigkeit zu entgegnen, wird seit einigen Jahren die Exploration von Methanhydratvorkommen intensiviert. Förderprojekte existieren bislang an der Ostküste zwischen Madras und Calcutta sowie im Meer von Andaman, zwischen Indien und Myanmar (Collett 2003). Die innerhalb des Indian Government's National Gas Hydrate Program im August 2006 abgeschlossene 113-tägige Forschungsreise hat ermutigende Ergebnisse bei der Erbohrung von Gashydraten geliefert und verdeutlicht die Ambitionen Indiens bei der Erschließung ihrer GashydratVorkommen. Ähnlich wie China konnte auch Indien kürzlich große Methanhydratvorkommen nachweisen. Beispielsweise wurde im Krishna-Godavari-Becken eine rund 130 Meter dicke Schicht Methanhydrat entdeckt (Collett et al. 2006).

Japan hat in den vergangenen fünf Jahren mehr in die Methanhydratforschung investiert als alle anderen Staaten zusammen. Das Hauptinteresse galt zu Beginn den Hydratvorkommen des Nankai Beckens östlich der japanischen Küste (Takahashi und Tsuji 2005). Das bereits bis 2003 durch das japanische Ministry of Economy, Trade and Industry sowie die Japan National Oil Company für die Methanhydratforschung bereitgestellte Budget belief sich auf rund 100 Millionen Dollar (Research Consortium for Methane Hydrate Resources in Japan 2008). Weitere rund 68 Millionen Dollar wurden im Zeitraum 2006/2007 für Forschungsaktivitäten in der See von Kumano investiert. Japan ist zudem finanziell maßgeblich an dem oben genannten Forschungsprojekt Mallik in Kanada beteiligt (Technology Research Center Japan National Oil Corporation 2003). Nachdem dort im Winter 2006/2007 ein Produktionstest erfolgreich verlaufen ist, wird Japan voraussichtlich weitere rund 75 Millionen Kanadische Dollar für Produktionstest in Kanada ausgeben (Federal Methane Hydrate Advisory Committee 2007). Perspektivisch sollen mit dem 2001 verabschiedeten und bis 2016 laufenden dreistufigen Japan's Methane Hydrate Exploitation Program alle notwendige Arbeiten abgeschlossen sein, so dass ab 2017 eine Förderung der marinen Gashydratvorkommen vor der Pazifikküste Japans sowie im Nankai Becken erfolgen kann (Research Consortium for Methane Hydrate Resources in Japan 2008). 
Viel versprechende Resultate im Rahmen ihres im Jahre 2005 eingeführten und auf 10 Jahre angelegten Programms zur Förderung von Gas aus Hydraten, vermeldete Anfang 2008 auch Südkorea (Park et al. 2008). Im November 2007 wurde die Ulleuung Basin Gas Hydrate Expedition I an der Ostküste Südkoreas erfolgreich abgeschlossen. Im Zuge dessen konnten im Rahmen von Bohrungen drei unterschiedliche Lagerstätten von Gashydraten lokalisiert werden. Eine detaillierte Auswertung dieser Probebohrungen ist derzeit noch nicht publiziert. Jedoch ist Südkorea - nach Japan weltweit der zweitgrößte Importeur von Flüssiggas zweifelsohne auf dem Weg, sich in diesem Kontext als eine der führenden Nationen zu etablieren. Von der bis 2015 anvisierten Förderung von Methanhydraten vor ihrer Ostküste ist für Südkorea ein entscheidender Schritt weg von einer derzeitig starken Importabhängigkeit ihrer Energieversorgung zu erwarten (U.S. Department of Energy 2007).

Weitere Länder mit bestehenden Methanhydrat-Forschungsprogrammen sind Kanada, Chile, Russland, Deutschland und Neuseeland (IFM - GEOMAR 2007). Deutschland verfügt über keine eigenen Vorkommen an Gashydraten und Forschung zu Methanhydraten wurde in der Vergangenheit überwiegend als Grundlagenforschung betrieben. In Form des kürzlich angelaufenen SUGAR-Verbundprojektes wird erstmals ein anwendungsbezogenes Forschungsvorhaben gefördert, welches insbesondere Fragen der Exploration sowie des Abbaus von submarinen Gashydratvorkommen und des Transportes von Erdgas umfasst (Wallmann 2007).

\section{Möglichkeiten einer Kombination der Methangewinnung aus Hydraten mit der} Sequestrierung von Kohlendioxid

Bislang sind die politischen Maßnahmen zur Reduzierung des $\mathrm{CO}_{2}$-Ausstoßes noch wenig erfolgreich und es ist notwendig, die eingefahrenen Wege der Klimapolitik zwar nicht zu verlassen, aber doch zu erweitern (Freund 2005; IPCC 2007a; IEA 2007b). Im Zuge dessen sind Alternativen grundsätzlich vorbehaltlos zu diskutieren. Neben dem beachtlichen Potential als Energieressource bietet die Nutzung von Methanhydraten ebenfalls eine aus Aspekten des Klimaschutzes hoch interessante Option: Die Kopplung des Methanabbaus mit der gleichzeitigen Sequestrierung von $\mathrm{CO}_{2}$.

Mit der $\mathrm{CO}_{2}$-Sequestrierung (bzw. Carbon Capture and Sequestration) wird nachfolgend eine Möglichkeit einbezogen, die das Ziel verfolgt, das bei der Nutzung fossiler Energieträger freigesetzte $\mathrm{CO}_{2}$ wieder aufzufangen bzw. temporär zu speichern, zu einer Lagerstätte zu transportieren und dort dauerhaft zu deponieren (Freund 2005; Dooley et al. 2006). 
Um die Methanhydrate kontrolliert abbauen zu können, wird hierbei die Destabilisierung der Methanhydrate durch die Injektion von flüssigem $\mathrm{CO}_{2}$ herbeigeführt (Seo und Lee 2001; Lee et al. 2003; Park et al. 2006; Kvamme et al. 2007). Im Zuge dessen entsteht Methangas und das zugefügte $\mathrm{CO}_{2}$ wird - bei geeigneten Druck-Temperatur-Bedingungen - in Form von Kohlendioxidhydraten in den ursprünglichen Methanhydratlagerstätten dauerhaft fixiert (Kvamme et al. 2007). Bei diesem Vorgehen wird zunächst flüssiges $\mathrm{CO}_{2}$ in die Lagerstätten des Methanhydrates injiziert. Dadurch werden die Methanhydrate zersetzt, Methangas kann gefördert werden und die Lagerstätten werden gleichzeitig mit Kohlendioxidhydraten aufgefüllt. Im Zuge dessen kann zudem eine teilweise Substitution von Methan durch $\mathrm{CO}_{2}$ erfolgen (Sloan 1998b; Bialas et al. 2007).

Den grundlegenden Ablauf des Zusammenspiels des hier beschriebenen Abbaus von Methanhydraten und der $\mathrm{CO}_{2}$-Sequestrierung skizziert Abbildung 2. Hierbei ist $\mathrm{zu}$ berücksichtigen, dass dieser Ansatz noch nie im industriellen Maßstab durchgeführt wurde. Die Erforschung der geochemischen Prozesse steht erst am Anfang und die bisherigen Kenntnisse beschränken sich auf konzeptionelle Studien bzw. experimentelle Laborarbeiten.

\begin{tabular}{|c|}
\hline Verbrennung fossiler Energieträger \\
\hline 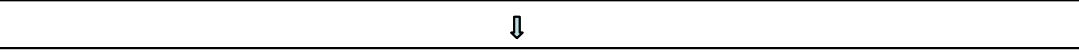 \\
\hline Speicherung des freigesetzten $\mathrm{CO}_{2}$ \\
\hline 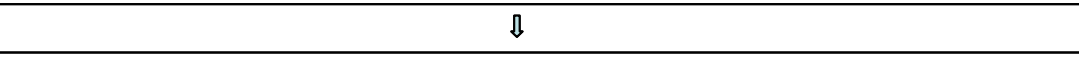 \\
\hline Transport des $\mathrm{CO}_{2}$ zur Lagerstätte \\
\hline$\sqrt{2}-19$ \\
\hline Injektion von flüssigem $\mathrm{CO}_{2}$ zur Destabilisierung der Methanhydrate \\
\hline$\sqrt{1}$ \\
\hline $\begin{array}{l}\text { Speicherung des austretenden Methangases und Deponierung von } \mathrm{CO}_{2} \\
\text { als Hydrat }\end{array}$ \\
\hline$\sqrt{1}$ \\
\hline Transport des Methangases \\
\hline 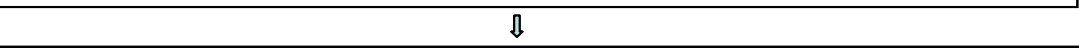 \\
\hline Nutzung des Methangases als Energieträger \\
\hline
\end{tabular}

Abb. 2: Ablaufschema der Kombination des Abbaus von Methanhydraten mit der $\mathrm{CO}_{2-}$ Sequestrierung. Quelle: Eigene Darstellung.

Aus klimapolitischer Perspektive ist daher ein weiteres Argument für den Abbau von Methanhydraten und die gleichzeitige Sequestrierung von Kohlendioxid einzubeziehen: Gegenüber Methanhydraten sind die deponierten Kohlendioxidhydrate innerhalb eines größeren Temperaturbereichs stabil (Wallmann 2007). Buffett und Archer (2004) haben gezeigt, dass über $80 \%$ der weltweiten Gashydratvorkommen schmelzen würden, wenn sich 
der Meeresboden um $3^{\circ} \mathrm{C}$ erwärmt. Wenn es möglich wäre, die oben beschriebene Technik ökonomisch vertretbar und sicher anzuwenden, besteht die Möglichkeit, die relativ temperatursensitiveren Methanhydrate vor einer Destabilisierung durch eine im Zuge des Klimawandels forcierte globale Erwärmung als Energieträger zu nutzen und die mit einer natürlichen Gasfreisetzung verbundenen Risiken zu reduzieren. Sofern von den zusätzlichen Emissionen durch Abbau, Umwandlung und Transport abstrahiert wird, würde die Ausbeutung der Methanhydratvorkommen grundsätzlich zu keiner weiteren Verstärkung des Treibhauseffektes beitragen, sondern vielmehr die Option einer dauerhaften Deponierung von $\mathrm{CO}_{2}$ eröffnen (Ohgaki et al. 1996; Seo und Lee 2001).

Hinsichtlich der Möglichkeiten einer Deponierung des $\mathrm{CO}_{2}$ ist grundsätzlich zwischen der Lagerung im Meeresboden und einer Deponierung an Land $\mathrm{zu}$ unterscheiden (Benson und Cook 2005; Caldeira und Akai 2005; Wallmann 2007). Für eine erste Abwägung zwischen diesen Optionen ist ein Trade-of zwischen den damit verbundenen Kosten und der langfristigen Sicherheit der Lagerstätten zu berücksichtigen (Herzog und Smekens 2005).

So ist die Lagerung in ausgebeuteten ehemaligen Gas- bzw. Öllagerstätten an Land als die kostengünstigere aber auch unsicherere Alternative anzusehen. Diese Lagerstätten sind relativ einfach zu erreichen und in vielen Fällen besteht sehr wahrscheinlich die Möglichkeit, auf einer bestehenden Infrastruktur aufzubauen. Dem stehen große Risiken gegenüber, da Fragen der Lagersicherheit hier noch weitestgehend unerforscht sind (Benson und Cook 2005). Kohlendioxid kann - bedingt durch entsprechende Temperatur-Druck-Bedingungen - in diesen Lagerstätten ausschließlich als mobiles Gas bzw. superkritisches Fluid deponiert werden. Demzufolge ist das Auftreten von Leckagen und im Zuge dessen ein Austreten des $\mathrm{CO}_{2}$ in die Atmosphäre ebenso wie eine Beeinflussung der Grundwasserqualität wahrscheinlich (Wells et al. 2006; Bialas et al. 2007). Gänzlich unbekannt sind derzeit noch die Gefahren einer Beeinträchtigung der Lagerstätten durch geologische Eingriffe und Naturkatastrophen in ihrer unmittelbaren Umgebung (Benson und Cook 2005; Wallmann 2007).

Die Deponierung von Kohlendioxid in Lagerstätten am Meeresboden stellt eine hohe Lagersicherheit in Aussicht, wobei gleichzeitig wesentlich höhere Kosten zu erwarten sind (Caldeira und Akai 2005; Wallmann 2007). Hier kann bereits auf erste Erkenntnisse zurückgegriffen werden, die eine sichere Deponierung in Aussicht stellen (Seo und Lee 2001; House et al. 2006). Da flüssiges $\mathrm{CO}_{2}$ in Wassertiefen von über 3000 Metern eine höhere Dichte als das oberhalb der Lagerstätten befindliche Meerwasser aufweist, stellt die von House et al. (2006) vorgeschlagene Option der Deponierung von flüssigem $\mathrm{CO}_{2}$ in 
Sedimenten dieser Tiefe die derzeit mit der größten Lagersicherheit verbundene Möglichkeit dar. Gleichwohl sind die notwendigen Bohrungen in derartigen Wassertiefen mit noch nicht quantifizierbaren, aber zweifelsohne hohen Kosten verbunden (Wallmann 2007).

Mit der bereits erforschten Injektion von $\mathrm{CO}_{2}$ in Methanhydratlagerstätten in Tiefen von 400 bis 1000 Metern (Seo und Lee 2001) sind demgegenüber sehr wahrscheinlich geringere Bohrkosten verbunden. Durch den gleichzeitigen Abbau von Methangas aus Hydraten besteht zudem die Möglichkeit, dass die Kosten der $\mathrm{CO}_{2}$-Sequestrierung durch entsprechende Erträge kompensiert oder sogar überkompensiert werden können. Eine sowohl ökologisch als auch ökonomisch realisierbare und tragfähige Lösung stellt daher die Kombination des Abbaus von Methanhydraten durch die Injektion von flüssigem $\mathrm{CO}_{2}$ und der gleichzeitigen Deponierung dieses $\mathrm{CO}_{2}$ in Lagerstätten im Meeresboden und einer Wassertiefe von 400 bis 1000 Metern in Aussicht. Entsprechende Forschungsarbeiten $\mathrm{zu}$ einer konkreten Umsetzung dieses Ansatzes stehen derzeit jedoch noch am Anfang und erste Ergebnisse sind nicht vor Ende des Jahres 2010 zu erwarten (Bialas et al. 2007; Wallmann 2007).

\section{Fazit und Ausblick}

Methanhydrate sind auch auf der Grundlage konservativer Schätzungen die im größten Umfang vorhandene Kohlenstoffressource. Ihre im Vergleich zu Erdöl und Erdgas breite geographische Verteilung sowie ihr erhebliches Potential machen sie zu einem begehrten Rohstoff und die Kombination ihres Abbaus bei einer gleichzeitigen Sequestrierung von Kohlendioxid eröffnet darüber hinaus klimapolitische Optionen. Demgegenüber bestehen die Gefahren einer Forcierung des Treibhauseffektes im Fall einer unkontrollierten Freisetzung von Methan in die Atmosphäre und der Destabilisierung der ozeanischen Sedimente. Bisher sind zudem die technischen Schwierigkeiten bei der Extraktion des Methans sowie der $\mathrm{CO}_{2}$ Sequestrierung noch nicht gelöst und hinsichtlich valider Kosten-Nutzen-Abschätzungen besteht weiterhin elementarer Forschungsbedarf.

Das ökonomische Kalkül einer optimalen intertemporalen Nutzung nicht regenerativer Ressourcen lässt sich auf der Grundlage zentraler Arbeiten von Solow (1974) und Stiglitz (1974) skizzieren. Die so genannte Solow-Stiglitz-Effizienzbedingung besagt, dass aus gesellschaftlicher Perspektive die optimale Verteilung des Vermögens auf Sachkapital und Ressourcen dann erreicht ist, wenn die Wachstumsrate der Grenzproduktivität der abgebauten Ressource der Grenzproduktivität des Kapitals entspricht. Dieser Ansatz ist bei einer praktischen Bewertung zumindest um Extraktionskosten sowie die erwarteten Schadenskosten der Ressourcennutzung und ihres Abbaus zu erweitern, da unterschiedliche Lagerstätten in 
der Regel nicht gleich gut zugänglich sind und ein Abbau von Methanhydraten sowohl mit unterschiedliche Extraktionskosten als auch einem unterschiedlichen Risiko verbunden ist. Eine derartige intertemporale Bewertung unterschiedlicher Methanhydratvorkommen ist jedoch bedingt durch nicht abschätzbare Kosten und nicht quantifizierbare Risiken derzeit nicht zu treffen.

Bislang sind für Aussagen zur zeitlichen und wirtschaftlichen Perspektive des Abbaus von Methanhydraten die oben beschriebenen nationalen Forschungsprogramme heranzuziehen. Durch ihre aktuellen Forschungsstrategien setzen insbesondere Japan, Südkorea und die USA die Maßstäbe für den Beginn einer kommerziellen Nutzung von Methan aus Methanhydraten. Im Zuge dessen ist entsprechend der Art der Lagerstätten grundsätzlich zwischen den folgenden drei Vorkommen zu differenzieren:

i) Methanhydrate in Permafrostregionen: Eine technische und wirtschaftliche Realisierbarkeit wird seitens der USA ab 2015 angestrebt.

ii) Marine Methanhydratvorkommen: Südkorea strebt eine Förderung ab 2015, Japan ab 2017, die USA ab 2025 an.

iii) Methanhydrate in Kontinentalhängen: Eine Förderung ist derzeit nicht absehbar.

Zusammenfassend ist zu erwarten, dass der Abbau von Methanhydraten je nach Art der Lagerstätte von Methanhydraten in Permafrostregionen über marine Methanhydratvorkommen bis hin zu Methanhydraten in Kontinentalhängen mit einem zunehmenden Risiko und steigenden Kosten verbunden ist. Für letztere existieren noch keine Förderkonzepte und ihre industrielle Nutzung ist derzeit als nicht realisierbar anzusehen.

Folglich sind sowohl die in den Permafrostgebieten als auch in Küstennähe lagernden Methanhydratvorkommen als zukünftige Ressourcen relevant; vor allem in Verbindung mit konventionellen Gaslagerstätten. Im Zuge einer industriellen Nutzung von Gas aus Methanhydraten kann sich Gas $\mathrm{zu}$ dem zentralen fossilen Energieträger entwickeln. Hinsichtlich der sich daraus ergebenden Auswirkungen auf die internationalen Beziehungen sind aus energiepolitischer Sicht neue Machtverhältnisse und Strukturen zu erwarten, wobei insbesondere Länder wie Indien, Japan, China, Südkorea und die USA durch ihre weit vorangeschrittenen Forschungsaktivitäten bzw. Methanhydratvorkommen einerseits ihre Abhängigkeit von Energieimporten senken als gleichermaßen zukünftig zu bedeutenden potentiellen Anbietern werden können. Die bedeutendste Nutzungsform des Methans aus Methanhydraten wird sehr wahrscheinlich - äquivalent zur Erdgasnutzung - die Integration in die bestehende Erdgasinfrastruktur. Zusätzlich wird aus Methanhydraten gewonnenes 
Methangas auch als Treibstoff attraktiv werden. Wie stark diese Auswirkungen sein werden und auf welchen Zeitskalen sie letztlich ablaufen, ist derzeit schwer zu prognostizieren, doch die zu erwartenden Weiterentwicklungen auch politisch zu verfolgen ist das Gebot der Stunde und notwendige Bedingung einer realitätsnahen wie zukunftsfähigen Energiepolitik.

Eine unvoreingenommen zu diskutierende Frage ist zudem der Umgang mit zukünftigen Möglichkeiten der Sequestrierung von Treibhausgasen - vor allem in Lagerstätten, aus denen vorher Methan aus Hydraten gefördert wurde. Grundsätzlich konnte in Laborexperimenten gezeigt werden, dass eine Substitution von Methan durch $\mathrm{CO}_{2}$ möglich ist (Ohgaki et al. 1996; Seo und Lee 2001). Jedoch stehen die Forschungsarbeiten diesbezüglich noch am Anfang. Bevor verlässliche Aussagen zur praktischen Implementierbarkeit und den damit verbundenen Kosten und Risiken getroffen werden können, gilt es die Ergebnisse weiterer anwendungsbezogener Erprobungen abzuwarten.

Bislang sind die Kosten einer alleinigen $\mathrm{CO}_{2}$-Sequestrierung nur grob abzuschätzen und bewegen sich in einer Größenordnung von 14 bis 91 US-Dollar pro vermiedener Tonne $\mathrm{CO}_{2}$, was einen Preisanstieg zwischen 21 und 91\% nach sich ziehen würde (Rubin et al. 2005). Im diesem Kontext eröffnet die Kombination der Nutzung von Methanhydraten mit der Sequestrierung von $\mathrm{CO}_{2}$ ein ökonomisches Potential, wenn für das deponierte Kohlendioxid (je nach rechtlicher Ausgestaltung) keine oder anteilig geringere Emissionsrechte gekauft bzw. gehalten werden müssen. Je nach Ausmaß des zukünftig zu erwartenden Preisanstiegs der Zertifikate im Zuge ihrer Versteigerung und Verknappung kann sich die Wirtschaftlichkeit dieses Ansatzes in den kommenden 10 bis 20 Jahren erheblich verbessern.

Es ist deutlich geworden, dass die Nutzung von Methanhydraten als Energieträger sowie die Kombination dieser Nutzung mit der Sequestrierung von Kohlendioxid noch einen grundlegenden anwendungsbezogenen Forschungsbedarf aufweist. Somit können am Ende dieses Beitrags noch keine konkreten Handlungsempfehlungen stehen. Vielmehr gilt es unter anderem die folgenden - über die aktuellen naturwissenschaftlich sowie technisch geprägten Forschungsansätze hinausgehenden - sozioökonomischen, juristischen und politischen Forschungsfragen aufzuwerfen:

- Wie können Methanhydrate und ihre Nutzung Eingang in neue Klimaschutzabkommen finden?

- Wie sind mögliche Methanfreisetzungen auf die nationalen Emissionsinventare anzurechnen?

- Welche Kosten sind mit dem Abbau von Methanhydraten aus unterschiedlichen Lagerstätten verbunden? 
- Welche technischen Möglichkeiten zum Abbau und zum Transport von aus Hydraten gewonnenem Methangas können zu welchen Kosten realisiert werden?

- Wie sind Risiken des Abbaus und des Transports von Methanhydraten monetär zu bewerten?

- Welche Kriterien sind an eine Umweltverträglichkeitsprüfung für den Abbau von Methanhydraten zu stellen?

- Welcher globale Einfluss zur Reduzierung des Klimawandels kann von einer Kombination der Nutzung von Methanhydraten und der $\mathrm{CO}_{2}$-Sequstrierung tatsächlich ausgehen?

- Welche Mindeststandards und Sicherheitsanforderungen sind für $\mathrm{CO}_{2}$-Lagerstätten verbindlich festzuschreiben, um einen dauerhaften Schutz auch vor Naturkatastrophen (wie beispielsweise dem verehrenden Erdbeben in China im Mai 2008) zu gewährleisten?

- Unter welchen ökonomischen Rahmenbedingungen ist die $\mathrm{CO}_{2}$-Sequestrierung ein wirtschaftliches Instrument zum Klimaschutz?

Des Weiteren besteht über die bislang dominierenden ökonomisch-technischen und klimapolitischen Betrachtungen hinaus ein essentieller Forschungsbedarf hinsichtlich der mit einem submarinen Methanhydratabbau verbundenen ökologischen Risiken. Der Ozeanboden ist ein sehr reiches Habitat, das bis jetzt relativ wenig vom Menschen beeinflusst wurde. Ein großflächiger Abbau von Methanhydraten würde mit hoher Wahrscheinlichkeit starke Folgen für diese Ökosysteme haben. Es muss daher sichergestellt werden, dass die drängenden energiepolitischen und ökonomischen Herausforderungen nicht dazu führen, dass die notwendige Behutsamkeit im Umgang mit der marinen Ökologie unberücksichtigt bleibt.

Darüber hinaus sind im Rahmen internationaler Projekte zur $\mathrm{CO}_{2}$-Sequstration rechtliche Fragen noch weitestgehend ungeklärt (Rubin et al. 2005). Einen ersten Einblick in diese Fragestellungen liefert eine Übersichtsarbeit des National Energy Technology Laboratory (2006). Zudem existieren noch keine tragfähigen Untersuchungen zur öffentlichen Akzeptanz und zur politischen Durchsetzbarkeit der $\mathrm{CO}_{2}$-Sequestrierung (Freund 2005).

Eine Beantwortung dieser Fragen in den kommenden fünf bis zehn Jahren wird zeigen, welche Rolle eine Nutzung von Methanhydraten für die zukünftige globale Energieversorgung tatsächlich spielen kann. Ein großes Potential ist Methanhydraten - vor allem in Kombination mit einer $\mathrm{CO}_{2}$-Sequestrierung - zweifelsohne $\mathrm{zu}$ attestieren. Mittlerweile wird die $\mathrm{CO}_{2}$-Sequestrierung auch innerhalb der EU als Klimaschutzmaßnahme diskutiert und am 23. Januar 2008 wurde ein Vorschlag für eine Richtlinie über die 
geologische Speicherung von Kohlendioxid vorgelegt (Kommission der Europäischen Gemeinschaften 2008).

Es wäre fahrlässig, diese Möglichkeit vor dem Hintergrund der entscheidenden energiepolitischen Ziele - Versorgungssicherheit und Klimaschutz - nicht zumindest solange $\mathrm{zu}$ verfolgen, bis eine fundierte Bewertung ihrer praktischen Potentiale und Risiken abgeschlossen ist.

\section{Literatur}

Archer, D. 2005. Destabilization of methane hydrates: a risk analysis. Externe Expertise für das WBGU-Sondergutachten „Die Zukunft der Meere - zu warm, zu hoch, zu sauer“. www.wbgu.de/wbgu_sn2006_ex01.pdf (abgerufen 01.09.2009).

Benson, S. und Cook, P. 2005. Underground geological storage. IPCC special report on carbon dioxide capture and storage. IPCC, Cambridge University Press: 195-276.

Bialas, J., Hensen, C. und Weinrebe, W. 2007. SUGAR-A: Submarine Gashydrat-Lagerstätten als Deponie für die CO2-Sequestrierung - Prospektion und Exploration. Antrag an das Bundesministerium für Bildung und Forschung. http://www.ifmgeomar.de/fileadmin/ifm-geomar/fb2/fb2_fe1/cutecht/SUGAR/Leitantrag_A.pdf (abgerufen 29.08.2009).

BP - British Petroleum 2007. BP Statistical Review of World Energy June 2007. London: BP.

Buffett, B. A. 2000. Clathrate hydrates. Annual Review of Earth and Planetary Sciences 28: 477-507.

Buffett, B. und Archer, D. 2004. Global inventory of methane clathrate: sensitivity to changes in the deep ocean. Earth and Planetary Science Letters 227 (3-4): 185-199.

Caldeira, K. und Akai, M. 2005. Ocean storage. IPCC special report on carbon dioxide capture and storage. IPCC, Cambridge University Press: 279-317.

Carroll, J. J. 2003. Natural gas hydrates: a guide for engineers. Amsterdam: Gulf Professional Publishers.

Collett, T. S. 2003. Natural gas hydrate as a potential energy resource. In: Natural gas hydrate in oceanic and permafrost environments. Herausgegeben von M. D. Max. Dordrecht, Boston, London: Kluwer Academic Publishers. 123-136.

Collett, T. S., Riedel, M., Boswell, R., Cochran, J. R., Kumar, P., Sethie, A. K., Sathe, A. V und NGHP Expedition-01 Scientific Party. Fire in the ice: Methane Hydrate Newsletter Fall 2006: 1-4. 
Dallimore, S. 2007. Community update on the 2006-2008 JOGMEC/NRCan/Aurora Malli Gas Hydrate Production Research Program, Northwest Territories, Canada. Fire in the ice: Methane Hydrate Newsletter Spring/Summer 2007: 6-7.

Dickens, G. R. 2004. Methane hydrate and abrupt climate change. Geotimes, November: 1822.

Dooley, J. J., Dahowski, R. T., Davidson, C. L., Wise, M. A., Gupta, N., Kim, S. H. und Malone, E. L. 2006. Carbon dioxide capture and geological storage: a core element of a global energy technology strategy to address climate change. A technical report from the second phase of the global energy technology strategy program. Battelle, Joint Global Climate Research Institute.

Driscoll, N. W., Weissel, J. K. und Goff, J. A. 2000. Potential for large-scale submarine slope failure and tsunami generation along the US mid-Atlantic coast. Geology 28 (5): $407-$ 410, 2000.

EIA - Energy Information Administration. 2007. Annual energy outlook 2007. http://www.eia.doe.gov/oiaf/archive/aeo07/pdf/0383(2007).pdf (abgerufen 20.07.2009).

Federal Methane Hydrate Advisory Committee. 2007. An assessment of the Methane Hydrate Research program and an assessment of the 5-Year Research Plan of the Department of Energy. Report to Congress. http://www.fe.doe.gov/programs/oilgas/hydrates/MHAC-07-ReportToCongressfinal.pdf (abgerufen 23.08.2009).

Freund, P. 2005. Introduction. IPCC special report on carbon dioxide capture and storage. IPCC, Cambridge University Press: 51-74.

Herzog, H. und Smekens, K. 2005. Cost and economical potential. IPCC special report on carbon dioxide capture and storage. IPCC, Cambridge University Press: 339-362.

Hornbach, M. J., Saffer, D. M. und Holbrook, W. S. 2004. Critically pressured free-gas reservoirs below gas-hydrate proavinces. Nature 427 (6970): 142-144.

House, K. Z., Schrag, D. P., Harvey C. F. und Lackner, K. S. 2006. Permanent carbon dioxide storage in deep-sea sediments, PNAS 103 (33): 12291-12295.

IEA - International Energy Agency. 2007a. World energy outlook 2005: China and India insights. Paris: International Energy Agency.

IEA - International Energy Agency. 2007b. CO2 Emissions from Fuel Combustion (detailed estimates) Vol 2007 release 01. 
http://oberon.sourceoecd.org/vl=1010473/cl=82/nw=1/rpsv/ij/oecdstats/16834291/v33 5n1/s1/p1 (abgerufen 29.08.2009).

IFM - GEOMAR. 2007. Gashydrate und Methankreislauf. http://www.gashydrate.de (abgerufen 28.08.2009).

IPCC - Intergovernmental Panel on Climate Change. 2007a. Climate change 2007: synthesis report. Contribution of working groups I, II and III to the Fourth Assessment Report of the Intergovernmental Panel on Climate Change. IPCC, Genf, Schweiz.

IPCC - Intergovernmental Panel on Climate Change. 2007b. Climate change 2007: the physical science basis. Contribution of working group I to the Fourth Assessment Report of the Intergovernmental Panel on Climate Change. IPCC, Genf, Schweiz.

Kennett, J. P., Cannariato, K. G. Hendy, I. L. und Behl, R. J. 2000. Carbon isotopic evidence for methane hydrate instability during quaternary interstadials, Science, 288 (5463): 128-133,

Kennett, J. P., Cannariato, K. G. Hendy, I. L. und Behl, R. J. 2003. Methane hydrates in quarternary climate change: the clathrate gun hypothesis. Washington D.C.: American Geophysical Union.

Kommission der Europäischen Gemeinschaften. 2008. Vorschlag für eine Richtlinie des Europäischen Parlaments und des Rates über die geologische Speicherung von Kohlendioxid. 2008/0015 (COD).

Kvamme, B., Graue, A., Buanes, T., Kuznetsova, T. und Ersland, G. 2007. Storage of $\mathrm{CO}_{2}$ in natural gas hydrate reservoirs and the effect of hydrate as an extra sealing in cold aquifers. International Journal of Greenhouse Gas Control 1: 236-246.

Kvenvolden, K. 1988. Methane hydrates and global climate. Global Biochemical Cycles 3: 221-229.

Kvenvolden, K. A. 2003. Natural gas hydrate: introduction and history of discovery. In: Natural gas hydrate in oceanic and permafrost environments. Herausgegeben von M. D. Max. Dordrecht, Boston, London: Kluwer Academic Publishers. 9-16.

Kvenvolden, K. A. und Lorenson, T. D. 2001. The global occurrence of natural gas hydrate. Geophysical Monograph 124: 87-98.

Lee, H., Seo, Y., Seo, Y.-T., Moundrakovski, I. L. und Ripmeester, J. A. 2003. Recovering methane from solid methane hydrate with carbon dioxide. Angewandte Chemie 115: 5202-5205.

Marland, G., Boden, T. A. und Andres, R. J. 2005. Global, regional, and national fossil fuel $\mathrm{CO}_{2}$ emissions. Carbon Dioxide Information Analysis Center, Oak Ridge National 
Laboratory, U.S. Department of Energy, Oak Ridge, Tenn., U.S.A.

http://cdiac.esd.ornl.gov/trends/emis/meth_reg.htm (abgerufen am 05.09.2009).

Max, M. D. und Pellenbarg, R. E. 2003. Introduction, physical properties, and natural occurrences of hydrate. In: Natural gas hydrate in oceanic and permafrost environments. Herausgegeben von M. D. Max. Dordrecht, Boston, London: Kluwer Academic Publishers. 1-8.

Max, M. D., Johnson, A. H. und Dillion, W. P. 2006. Economic geology of natural gas hydrate. Dordrecht: Springer.

Milkov, A. V. 2004. Global estimates of hydrate-bound gas in marine sediments: how much is really out there? Earth-Science Reviews 66 (3-4): 183-197.

National Energy Technology Laboratory 2006. International carbon capture and storage projects overcoming legal barriers. DOE/NETL-2006/1236.

National Energy Technology Laboratory. 2007. Program facts - methane hydrates. www.netl.doe.gov/scngo (abgerufen am 01.09.2009).

Ohgaki, T., Takano, H., Sangawa, H., Matsubara, T. und Nakano, S. 1996. Methane exploitatiom by carbon dioxide from gas hydrates - phase equilibria for $\mathrm{CO} 2-\mathrm{CH} 4$ mixed hydrate systems. Journal of Chemical Engineering of Japan 29: 478-483.

Park, Y., Kim, D.-Y., Lee, J.-W., Huh, D.-G., Park, K.-P., Lee, J. und Lee, H. 2006. Sequestration carbon dioxide into complex structures of naturally occurring gas hydrates. PNAS 103: 12690-12694.

Park, K.-P., Bahk, J.-J., Kwon, Y., Kim, G. Y., Riedel, M., Holland, M., Schultheiss, P., Roe, K. und The UBGH-I Scientific Party. 2008. Korean national program expedition confirms rich gas hydrate deposits in the Ulleung Basin, East Sea. Fire in the ice: Methane Hydrate Newsletter Spring 2008: 6-9.

Research Consortium for Methane Hydrate Resources in Japan. 2008. Background and organization. www.mh21japan.gr.jp/english/mh21-2.html (abgerufen 23.08.2009).

Rogner, H.-H. 1997. An assessment of world hydrocarbon resources. Annual Review of Energy and the Environment 22: 217-262.

Rubin, E., Meyer, L. und De Coninck, H. 2005. Technical summary. IPCC special report on carbon dioxide capture and storage. IPCC, Cambridge University Press: 17-50.

Seo, Y-T. und Lee, H. 2001. Multiple-phase hydrate equilibria of the temary carbon dioxide, methane, water mixtures. The Journal of Physical Chemistry B: 10084-10090.

Sloan, E. D. 1998a. Gas hydrates: review of physical/chemical properties. Energy \& Fuels 12: 191-196. 
Sloan, E. D. 1998b. Clathrate hydrates of natural gases. Marcel Decker Inc.

Solow, R. M. 1974. The economics of resources or the resources of economics. American Economic Review 64: 1-14.

Stern, N. et al. 2006. Stern review: the economics of climate change. London: HM Treasury.

Stiglitz, J. E. 1974. Growth with exhaustible natural resources: efficient and optimal growth paths. Review of Economic Studies 41, Symposium on the Economics of Exhaustible Resources: 123-137.

Takahashi, H. und Tsuji, Y. 2005. Japan explores for hydrate in the Nankai Trough. Oil \& Gas Journal 103: 48-53.

Technology Research Center Japan National Oil Corporation. 2003. From Mallik to the future. Program \& abstracts of the Mallik International Symposium in Makuhari. http://www.mh21japan.gr.jp/mallik2002/pdf/1226/Mallik\%20Abstract.pdf (abgerufen 23.08.2009).

Torres, M. E., Wallmann, K., Trehu, A. M., Bohrmann, G., Borowski, W. S. und Tomaru, H. 2004. Gas hydrate growth, methane transport, and choride enrichment in the southern summit of Hydrate Ridge, Cascadia margin off Oregon. Earth and Planetary Science Letters 226: 225-241.

U.S. Department of Energy. 2006. An interagency roadmap for methane hydrate research and development. http://www.netl.doe.gov/technologies/oilgas/publications/Hydrates/pdf/InteragencyRoadmap.pdf (abgerufen am 23.09.2009).

U.S. Department of Energy. 2007. Early results from Korean gas hydrate research effort are encouraging. Fire in the ice: Methane Hydrate Newsletter Fall 2007: 12.

USGS - USGS Science Center for Coastal and Marine Geology. 2006. Gas hydrate studies. www.woodshole.er.usgs.gov/project-pages/hydrates/index.html (abgerufen 01.09.2009).

Wallmann, K. 2007. Submarine Gashydrat-Lagerstätten: Erkundung, Abbau und Transport (SUGAR). Antrag an das Bundesministerium für Wirtschaft und Technologie. http://www.ifm-geomar.de/fileadmin/ifmgeomar/fb2/fb2_fe1/cutecht/SUGAR/Leitantrag_B.pdf (abgerufen 23.08.2009).

Wallmann, K., Aloisi, G., Haechel, M., Obzhirov, G., Pavlova, G. und Tishchenko, P. 2006. Kinetics of organic matter degradation, microbial methane generation, and gas hydrate formation in anoxic marine sediments. Geochimica et Cosmochimica Acta 70: 39053927. 
WBGU - Wissenschaftlicher Beirat der Bundesregierung Globale Umweltveränderungen. 2006. Die Zukunft der Meere - zu warm, zu hoch, zu sauer. WBGU-Sondergutachten 2006. Berlin.

Wells, A. W., Hammack, R. W., Veloski, G. A., Diekl, J. R. und Strazisar, B. R. 2006. Monitoring, miigation, and verification at sequestration sites: SEQURE technologies and the challenge for geophysical detection. The leading edge 25: 1264-1270.

World Energy Council. 2000. World energy assessment: energy and the challenge of sustainability. New York: United Nations Development Programme.

World Energy Council. 2007. 2007 Survey of Energy Resources. London: World Energy Council.

World Resources Institute. 2006. Climate analysis indicators tool. Washington, DC: World Resources Institute. http://cait.wri.org (abgerufen 04.08.2009).

Zhang, H., Yang, S., Wu, N., Schultheiss, P. und GMGS-I Science Team. 2007a. China's first gas hydrate expedition successful. Fire in the ice: Methane Hydrate Newsletter Spring/Summer 2007: 1.

Zhang, H., Yang, S., Wu, N., Holland, M., Schultheiss, P., Rose, K., Butler, H., Humphrey, G. und GMGS-I Science Team. 2007b. Successful and surprising results for China's first gas hydrate drilling expedition. Fire in the ice: Methane Hydrate Newsletter Fall 2007: 6-9. 


\section{Working Paper Series in Economics}

(recent issues)

No.146: Sandra Derissen, Martin Quaas and Stefan Baumgärtner: The relationship between resilience and sustainable development of ecological-economic systems, October 2009

No.145: Anne-Kathrin Last und Heike Wetzel: Effizienzmessverfahren - Eine Einführung, September 2009

No.144: Horst Raff and Joachim Wagner: Intra-Industry Adjustment to Import Competition: Theory and Application to the German Clothing Industry, September 2009

No.143: Nils Braakmann: Are there social returns to both firm-level and regional human capital? - Evidence from German social security data. September 2009

No.142: Nils Braakmann and Alexander Vogel: How does economic integration influence employment and wages in border regions? The case of the EU-enlargement 2004 and Germany's eastern border, September 2009

No.141: Stefanie Glotzbach and Stefan Baumgärtner: The relationship between intra- and intergenerational ecological justice. Determinants of goal conflicts and synergies in sustainability policy. September 2009

No.140: Alexander Vogel: Exportprämien unternehmensnaher Dienstleister in Niedersachsen, September 2009

No.139: Alexander Vogel: Die Dynamik der Export- und Importbeteiligung niedersächsischer Industrieunternehmen im interregionalen Vergleich 2001-2006, September 2009

No.138: Stefan Baumgärtner and Martin F. Quaas: What is sustainability economics? September 2009

No.137: Roland Olbrich, Martin F. Quaas and Stefan Baumgärtner: Sustainable use of ecosystem services under multiple risks - a survey of commercial cattle farmers in semiarid rangelands in Namibia, September 2009

No.136: Joachim Wagner: One-third codetermination at company supervisory boards and firm performance in German manufacturing industries: First direct evidence from a new type of enterprise data, August 2009

No.135: Joachim Wagner: The Reasearch Potential of New Types of Enterprise Data based on Surveys from Official Statistics in Germany, August 2009

No.134: Anne-Kathrin Last and Heike Wetzel: The Efficiency of German Public Theaters: A Stochastic Frontier Analysis Approach, July 2009

No.133: Markus Groth: Das Conservation Reserve Program: Erfahrungen und Perspektiven für die europäische Agrarumweltpolitik, Juli 2009

No.132: Stefan Baumgärtner and Sebastian Strunz: The economic insurance value of ecosystem resilience, July 2009

No.131: Matthias Schröter, Oliver Jakoby, Roland Olbrich, Marcus Eichhorn and Stefan Baumgärtner: Remote sensing of bush encroachment on commercial cattle farms in semi-arid rangelands in Namibia, July 2009

No.130: Nils Braakmann: Other-regarding preferences, spousal disability and happiness: Evidence for German Couples, May 2009

No.129: Alexander Vogel and Joachim Wagner: Exports and Profitability - First Evidence for German Services Enterprises, May 2009 
No.128: Sebastian Troch: Drittelbeteiligung im Aufsichtsrat - Gesetzliche Regelung versus Unternehmenspraxis. Ausmaß und Bestimmungsgründe der Umgehung des Drittelbeteiligungsgesetzes in Industrieunternehmen, Mai 2009

No.127: Alexander Vogel: The German Business Services Statistics Panel 2003 to 2007, May 2009 [forthcoming in: Schmollers Jahrbuch 129 (2009)]

No.126: Nils Braakmann: The role of firm-level and regional human capital fort he social returns to education - Evidence from German social security data, April 2009

No.125: Elke Bertke und Markus Groth: Angebot und Nachfrage nach Umweltleistungen in einem marktanalogen Agrarumweltprogramm - Ergebnisse einer Pilotstudie, April 2009

No.124: Nils Braakmann and Alexander Vogel: The impact of the 2004 EU-enlargement on enterprise performance and exports of service enterprises in the German eastern border region, April 2009 [revised version forthcoming in: Review of World Economics]

No.123: Alexander Eickelpasch and Alexander Vogel: Determinants of Export Behaviour of German Business Services Companies, March 2009

No.122: Maik Heinemann: Stability under Learning of Equilibria in Financial Markets with Supply Information, March 2009

No.121: Thomas Wein: Auf der Speisekarte der DPAG: Rechtliche oder ökonomische Marktzutrittsschranken? März 2009

No.120: Nils Braakmann und Joachim Wagner: Product Diversification and Stability of Employment and Sales: First Evidence from German Manufacturing Firms, February 2009

No.119: Markus Groth: The transferability and performance of payment-by-results biodiversity conservation procurement auctions: empirical evidence from northernmost Germany, February 2009

No.118: Anja Klaubert: Being religious - A Question of Incentives? February 2009

No.117: Sourafel Girma, Holger Görg and Joachim Wagner: Subsidies and Exports in Germany. First Evidence from Enterprise Panel Data, January 2009

No.116: Alexander Vogel und Joachim Wagner: Import, Export und Produktivität in niedersächsischen Unternehmen des Verarbeitenden Gewerbes, Januar 2009

No.115: Nils Braakmann and Joachim Wagner: Product Differentiation and Profitability in German Manufacturing Firms, January 2009

No.114: Franziska Boneberg: Die Drittelmitbestimmungslücke im Dienstleistungssektor: Ausmaß und Bestimmungsgründe, Januar 2009

No.113: Institut für Volkswirtschaftslehre: Forschungsbericht 2008, Januar 2009

No.112: Nils Braakmann: The role of psychological traits and the gender gap in full-time employment and wages: Evidence from Germany. January 2009

No.111: Alexander Vogel: Exporter Performance in the German Business Services Sector: First Evidence from the Services Statistics Panel. January 2009 [revised version forthcoming in: The Service Industries Journal]

No.110: Joachim Wagner: Wer wird subventioniert? Subventionen in deutschen Industrieunternehmen 1999 - 2006. Januar 2009 
No.109: Martin F. Quaas, Stefan Baumgärtner, Sandra Derissen, and Sebastian Strunz: Institutions and preferences determine resilience of ecological-economic systems. December 2008

No.108: Maik Heinemann: Messung und Darstellung von Ungleichheit. November 2008

No.107: Claus Schnabel \& Joachim Wagner: Union Membership and Age: The inverted U-shape hypothesis under test. November 2008

No.106: Alexander Vogel \& Joachim Wagner: Higher Productivity in Importing German Manufacturing Firms: Self-selection, Learning from Importing, or Both? November 2008 [revised version forthcoming in: Review of World Economics]

No.105: Markus Groth: Kosteneffizienter und effektiver Biodiversitätsschutz durch Ausschreibungen und eine ergebnisorientierte Honorierung: Das Modellprojekt „Blühendes Steinburg“. November 2008

No.104: Alexander Vogel \& Joachim Wagner: Export, Import und Produktivität wissensintensiver KMUs in Deutschland. Oktober 2008

No.103: Christiane Clemens \& Maik Heinemann: On Entrepreneurial Risk - Taking and the Macroeconomic Effects Of Financial Constraints, October 2008

No.102: Helmut Fryges \& Joachim Wagner: Exports and Profitability - First Evidence for German Manufacturing Firms. October 2008

No.101: Heike Wetzel: Productivity Growth in European Railways: Technological Progress, Efficiency Change and Scale Effects. October 2008

No.100: Henry Sabrowski: Inflation Expectation Formation of German Consumers: Rational or Adaptive? October 2008

No.99: Joachim Wagner: Produktdifferenzierung in deutschen Industrieunternehmen 1995 2004: Ausmaß und Bestimmungsgründe, Oktober 2008

No.98: Jan Kranich: Agglomeration, vertical specialization, and the strength of industrial linkages, September 2008

No.97: Joachim Wagner: Exports and firm characteristics - First evidence from Fractional Probit Panel Estimates, August 2008

No.96: Nils Braakmann: The smoking wage penalty in the United Kingdom: Regression and matching evidence from the British Household Panel Survey, August 2008

No.95: Joachim Wagner: Exportaktivitäten und Rendite in niedersächsischen Industrieunternehmen, August 2008 [publiziert in: Statistische Monatshefte Niedersachsen 62 (2008), 10,552-560]

No.94: Joachim Wagner: Wirken sich Exportaktivitäten positiv auf die Rendite von deutschen Industrieunternehmen aus?, August 2008 [publiziert in: Wirtschaftsdienst, 88 (2008) 10, 690-696]

No.93: Claus Schnabel \& Joachim Wagner: The aging of the unions in West Germany, 1980-2006, August 2008 [forthcoming in: Jahrbücher für Nationalökonomie und Statistik]

No.92: Alexander Vogel and Stefan Dittrich: The German turnover tax statistics panels, August 2008

[published in: Schmollers Jahrbuch 128 (2008), 4, 661-670]

(see www.leuphana.de/vwl/papers for a complete list) 
Leuphana Universität Lüneburg

Institut für Volkswirtschaftslehre

Postfach 2440

D-21314 Lüneburg

Tel.: ++49 41316772321

email: brodt@leuphana.de

www.leuphana.de/vwl/papers 